ARTÍCULO ORIGINAL

\title{
EVALUACIÓN DE LA TOXICIDAD DE CHALCONAS SINTÉTICAS CON POTENCIAL ANTI-LEISHMANIA EN RATONES BALB/C
}

\author{
Karina Cancino (11,a,Inés Castro (11,b, Carlos Yauri(1,c, Valérie Jullian(102,d,

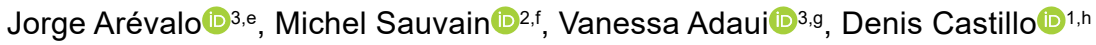 \\ 1 Laboratorio Mixto Internacional Andino-Amazónico de Química de la Vida IRD-UPCH, Universidad Peruana Cayetano \\ Heredia, Lima, Perú. \\ 2 UMR 152 PHARMA-DEV, Université de Toulouse, IRD, Toulouse, Francia. \\ 3 Laboratorio de Biología Celular y Molecular de Tripanosomátidos, Instituto de Medicina Tropical Alexander von Humboldt, \\ Universidad Peruana Cayetano Heredia, Lima, Perú.

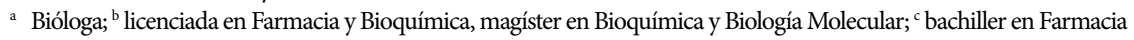 \\ y Bioquímica; ${ }^{d}$ química, $\mathrm{PhD}$ en Química Orgánica; ${ }^{\mathrm{e}}$ biólogo, doctor en Ciencias; ${ }^{\mathrm{f}}$ químico farmacéutico, $\mathrm{PhD}$ en \\ Farmacoquímica; ${ }^{\mathrm{g}}$ bióloga, $\mathrm{PhD}$ en Ciencias Biomédicas; ${ }^{\mathrm{h}}$ biólogo, magíster en Bioquímica y Biología Molecular.
}

\section{RESUMEN}

Objetivo: Evaluar la toxicidad de tres chalconas sintéticas administradas por vía intraperitoneal en ratones BALB/c. Materiales y métodos: La dosis letal media $\left(\mathrm{DL}_{50}\right)$ se estimó por el método Up-and-Down de Dixon. La toxicidad subcrónica de las chalconas se evaluó a 20 y $40 \mathrm{mg} / \mathrm{kg}$ por 21 días. Se evaluó el efecto tóxico a nivel de comportamiento, fisiológico, bioquímico e histológico. Resultados: La chalcona 43 generó moco en las heces, daño visceral (hígado) y alteración en el coeficiente de órganos (riñón, $\mathrm{p}=0,037$ y cerebro, $\mathrm{p}=0,008$ ) en comparación con el grupo control. Además, en el análisis histológico se observó que esta chalcona produjo edema, inflamación y necrosis en los órganos evaluados, aunque no hubo diferencia significativa con el control. Todos los parámetros bioquímicos no difirieron significativamente entre los grupos de tratamiento a dosis de $40 \mathrm{mg} / \mathrm{kg}$ y el control. Conclusiones: $\mathrm{La} \mathrm{DL}_{50}$ para las tres chalconas fue superior a $550 \mathrm{mg} / \mathrm{kg}$ de peso corporal. Las chalconas 40 y 42 son relativamente no tóxicas. Ambas pueden considerarse seguras para la aplicación vía intraperitoneal en ratones BALB/c $y$, en consecuencia, son posibles candidatas para ser usadas en el tratamiento contra las leishmaniosis.

Palabras clave: Chalcona; Desarrollo de Medicamentos; Ratones Endogámicos BALB C; Pruebas de Toxicidad Subcrónica; Dosificación Letal Mediana (fuente: DeCS BIREME).

\section{TOXICITY ASSESSMENT OF SYNTHETIC CHALCONES WITH ANTILEISHMANIAL POTENTIAL IN BALB/C MICE}

Citar como: Cancino K, Castro I, Yauri C, Jullian V, Arévalo J, Sauvain M, et al. Evaluación de la toxicidad de chalconas sintéticas con potencial anti-Leishmania en ratones BALB/c. Rev Peru Med Exp Salud Publica. 2021;38(3):42433. doi: https://doi.org/10.17843/ rpmesp.2021.383.6937.

Correspondencia:Vanessa Adaui; Centro de Investigación e Innovación, Facultad de Ciencias de la Salud, Universidad Peruana de Ciencias Aplicadas, Av. Alameda San Marcos cuadra 2, Lima 15067, Perú; vanessa. adaui@upc.edu.pe

Recibido: $18 / 12 / 2020$ Aprobado: 04/08/2021 En línea: 03/09/2021

\section{ABSTRACT}

Objective: To evaluate the toxicity of three synthetic chalcones administered intraperitoneally to BALB/c mice. Materials and methods: The median lethal dose $\left(\mathrm{LD}_{50}\right)$ was estimated by Dixon's Up-and-Down method. Subchronic toxicity of chalcones was evaluated at 20 and $40 \mathrm{mg} / \mathrm{kg}$ for 21 days. Behavioral, physiological, biochemical, biochemical and histological toxic effects were evaluated. Results: Chalcone 43 produced mucus in feces, visceral damage (liver) and alterations in organ coefficient (kidney, $\mathrm{p}=0.037$ and brain, $p=0.008$ ) when compared to the control group. In addition, histological analysis showed that this chalcone produced edema, inflammation and necrosis in the organs that were evaluated, although there was no significant difference with the control. None of the biochemical parameters differed significantly between the treatment groups at $40 \mathrm{mg} / \mathrm{kg}$ dose and the control. Conclusions: The $\mathrm{LD}_{50}$ for all three chalcones was greater than $550 \mathrm{mg} / \mathrm{kg}$ of body weight. Chalcones 40 and 42 were found to be relatively non-toxic. Both can be considered safe for intraperitoneal application in BALB/c mice and, consequently, are potential candidates for use in the treatment of leishmaniasis.

Keywords: Synthetic Chalcones; Drug Development; BALB/c Mice; Subchronic Toxicity; Median Lethal Dose (source: MeSH NLM).

\section{INTRODUCCIÓN}

Las chalconas son abundantes en plantas comestibles, forman parte de la familia de los flavonoides y cuentan con un amplio espectro de actividades farmacológicas ${ }^{(1,2)}$. En cuanto a 
su actividad antiparasitaria destaca su actividad contra protozoarios patógenos de la familia Trypanosomatidae que incluye los géneros Trypanosoma (causante de la enfermedad de Chagas y de la tripanosomiasis humana africana) y Leishmania (causante de leishmaniosis) ${ }^{(3,4)}$. Varios estudios reportan la síntesis química y evaluación de análogos de chalconas naturales con la finalidad de desarrollar compuestos con una actividad selectiva antitripanosomátida mejorada ${ }^{(3-5)}$, entre ellos, derivados de chalconas con prometedora actividad inhibitoria de la arginasa en Leishmania infantum ${ }^{(6)}$. Estos estudios muestran el esfuerzo constante de búsqueda de nuevas alternativas terapéuticas contra las tripanosomiasis y leishmaniosis, que afectan a millones de personas y animales en todo el mundo ${ }^{(7)}$, y para las cuales los pocos tratamientos disponibles presentan limitaciones ${ }^{(8)}$.

En estudios previos con una nueva serie de chalconas sintéticas, nuestro equipo evaluó la actividad in vitro contra especies de Leishmania responsables de leishmaniosis cutánea y mucosa del Nuevo Mundo. Nueve chalconas (entre ellas, 40, 42 y 43) presentaron actividad biológica y selectividad comparable a la anfotericina $\mathrm{B}$, tanto sobre amastigotes axénicos como amastigotes intracelulares en infecciones de macrófagos, con índices de selectividad de 10 a $80^{(9)}$. Se evaluaron cinco chalconas in vivo en ratones $\mathrm{BALB} / \mathrm{c}$ infectados por Leishmania amazonensis (empleando el modelo clásico de inoculación subcutánea del parásito en la almohadilla plantar). Dos de estos compuestos (42 y 43) administrados por vía intralesional mostraron una reducción de la carga parasitaria comparada con controles infectados no tratados ${ }^{(9)}$. Para dicha evaluación se administraron los compuestos en una dosis de $5 \mathrm{mg} / \mathrm{kg} /$ día (dosis casi 7 veces menor que el control positivo con glucantime, fármaco de referencia de primera línea, administrado a $33 \mathrm{mg} / \mathrm{kg} /$ día). En base a estos resultados y a fin de encaminar el desarrollo de un potencial nuevo fármaco antileishmanial, se decidió evaluar la toxicidad en modelos experimentales in vivo tomando en cuenta la vía de administración de los compuestos, dosis y esquema de tratamiento, similares a lo empleado en el tratamiento clínico con fármacos de referencia. La presente investigación tuvo como objetivo determinar la dosis letal media y la posible toxicidad subcrónica de tres chalconas sintéticas (40, 42 y 43) administradas por vía intraperitoneal en ratones $\mathrm{BALB} / \mathrm{c}$.

\section{MATERIALES Y MÉTODOS}

\section{Diseño}

Se realizó un estudio experimental con las tres chalconas (40, 42 y 43) identificadas previamente por nuestro equipo. Para la determinación de la $\mathrm{DL}_{50}$ se emplearon ratones hembra $(\mathrm{n}=6)$ por ser más susceptibles a los compuestos según la Organisation for Economic Cooperation and Development (OECD) ${ }^{(10)}$. Se estimó la $\mathrm{DL}_{50}$ utilizando el método Up-and-Down ${ }^{(11)}$. Para determinar

\section{MENSAJES CLAVE}

Motivación para realizar el estudio: Las chalconas tienen un amplio espectro de actividad antiparasitaria, por ello fueron evaluadas como tratamiento intralesional contra la leishmaniosis cutánea en un estudio previo. Aún no se ha descrito la toxicidad de estos compuestos aplicados por vía intraperitoneal en modelos in vivo.

Principales hallazgos: Las tres chalconas sintéticas evaluadas tuvieron una dosis letal media $\left(\mathrm{DL}_{50}\right)$ mayor a $550 \mathrm{mg} / \mathrm{kg}$ de peso. La chalcona 43 produce daño visceral y edema en los órganos internos a diferencia de las chalconas 40 y 42.

Implicancias: Las chalconas 40 y 42, relativamente no tóxicas, pueden formar parte de futuras estrategias terapéuticas contra las leishmaniosis.

la toxicidad subcrónica, se distribuyeron los animales aleatoriamente en ocho grupos (6 ratones macho por grupo). Se eligieron dos dosis por chalcona según la literatura revisada ${ }^{(4)}: 20 \mathrm{mg} / \mathrm{kg}$ (dosis A) y $40 \mathrm{mg} / \mathrm{kg}$ (dosis B) de peso del animal. Cada dosis se administró según el esquema de tratamiento: nueve administraciones en 21 días (tres por semana) en un volumen máximo de $100 \mu \mathrm{L}$ por vía intraperitoneal (IP). Se incluyeron el grupo control, al cual se le aplicó los solventes empleados en la disolución de los compuestos, y el grupo Sham, que no recibió tratamiento alguno. El diseño experimental para el registro de datos clínicos (peso corporal, coeficiente de órganos, mortalidad, bioquímica sanguínea e histopatología) se describe en la Figura 1.

\section{Procedimientos}

\section{Animales}

Se obtuvieron ratones BALB/c (8 - 10 semanas de edad, $30 \mathrm{~g}$ de peso y libres de patógenos) del Instituto Nacional de Salud de Perú. Se mantuvo el bioterio a $22{ }^{\circ} \mathrm{C} \pm 2{ }^{\circ} \mathrm{C}$ y $60 \% \pm 10 \%$ de humedad relativa con un ciclo de luz-oscuridad de $12 \mathrm{~h}$. Los animales recibieron alimento y agua ad libitum.

\section{Químicos}

La síntesis de novo de la chalcona $40\left(\mathrm{C}_{14} \mathrm{H}_{12} \mathrm{O}_{5}\right)$ se realizó siguiendo las metodologías descritas por Aponte et al. ${ }^{(3)} \mathrm{a}$ partir de la 2,4',6'-trihidroxiacetofenona, mientras que la chalcona $42\left(\mathrm{C}_{21} \mathrm{H}_{22} \mathrm{O}_{8}\right)$ y la chalcona $43\left(\mathrm{C}_{21} \mathrm{H}_{24} \mathrm{O}_{8}\right)$ se sintetizaron por una vía similar, descrita por Du et al. ${ }^{(12)}$.

La identidad y la pureza de los compuestos se verificaron por espectroscopía de resonancia magnética nuclear (RMN) (Figura 2). Los espectros se grabaron en el servicio de RMN de la Pontificia Universidad Católica del Perú. Para la aplicación por vía IP en los ratones, se disolvieron los compuestos en dimetilsulfóxido (DMSO) al 5\% y Tween 20 al 4\% en tampón fosfato salino (PBS) 0,01 M. 


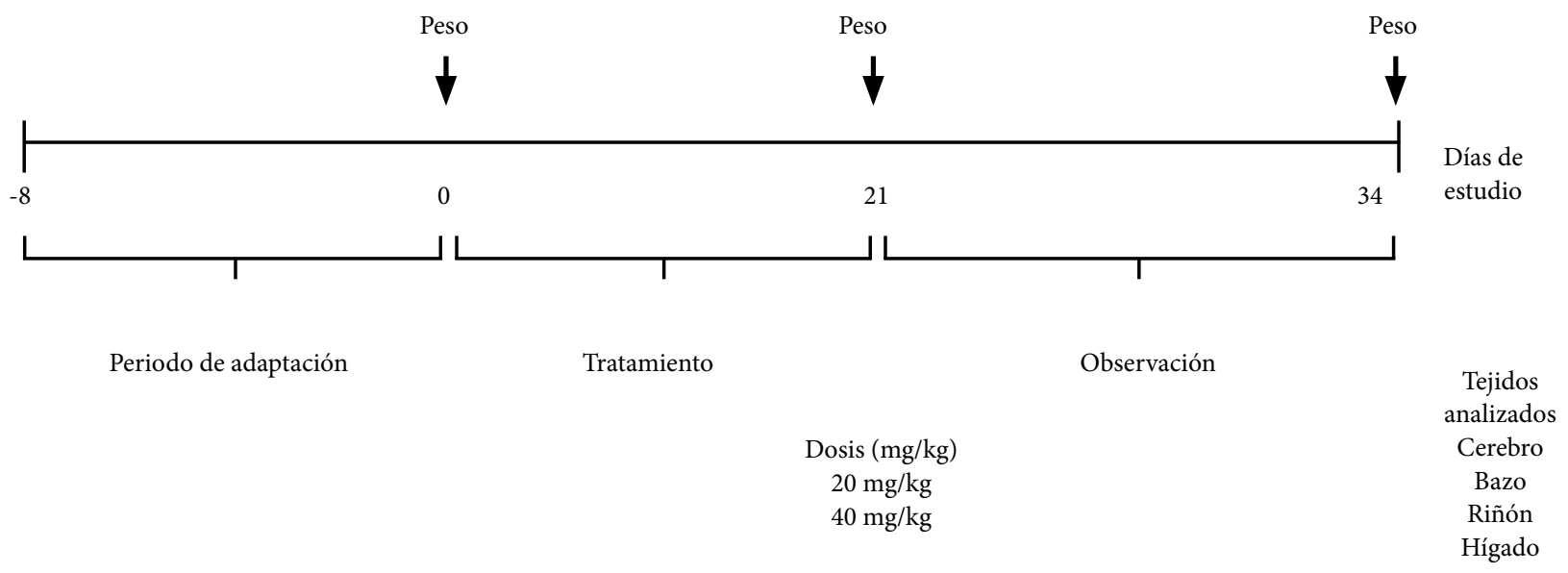

Figura 1. Diseño experimental del estudio de toxicidad subcrónica.

Estimación de la $\mathrm{DL}_{50}$ usando el método Up-and-Down de Dixon El primer animal recibe una dosis por debajo de la mejor estimación preliminar de la $\mathrm{DL}_{50}$ por vía oral ${ }^{(11)}$. Si el animal sobrevive, el segundo animal recibe una dosis más alta. Si el primer animal muere o aparece moribundo, el segundo animal recibe una dosis más baja. La dosis recomendada a utilizar cuando no existe conocimiento precedente es $175 \mathrm{mg} /$ kg de peso. Se inició el experimento con esta dosis y luego se emplearon $550 \mathrm{mg} / \mathrm{kg}$ de peso. Se evaluó al animal 48 horas luego de cada dosis. Durante ese periodo (a los 15 minutos, 30 minutos, 24 horas y 48 horas) se aplicó el test de Irwin modificado ${ }^{(13)}$. Para el examen macroscópico del hígado, bazo y riñón, se eutanasiaron los animales seis días después de la administración de las dosis.<smiles>COc1cc(O)cc(O)c1C(=O)/C=C/c1ccco1</smiles>

(2E)-1-(2,4-Dihydroxy-6-methoxyphenyl)-3-(2-fura nyl)-2-propen-1-one Fórmula molecular: $\mathrm{C}_{14} \mathrm{H}_{12} \mathrm{O}_{5}$ Peso molecular: $260,24 \mathrm{~g} / \mathrm{mol}$<smiles>COCOc1cc(OC)c(C(=O)/C=C/c2ccc3c(c2)OCO3)c(OCOC)c1</smiles>

(2E)-3-(1,3-Benzodioxol-5-yl)-1-(2,4-dimethoxymet hyl-6-methoxyphenyl)-2-propen-1-one Fórmula molecular: $\mathrm{C}_{21} \mathrm{H}_{22} \mathrm{O}$ Peso molecular: $402,39 \mathrm{~g} / \mathrm{mol}$<smiles>COCOc1cc(O)c(C(=O)/C=C/c2cc(OC)c(OC)c(OC)c2)c(OC)c1</smiles>

(2E)-1-(2-Hydroxy-4-methoxymethyl-6-methoxyphenyl)-3

-(3,4,5-trimethoxyphenyl)-2-propen-1-one

Fórmula molecular: $\mathrm{C}_{21} \mathrm{H}_{24} \mathrm{O}_{8}$

Peso molecular: $404,41 \mathrm{~g} / \mathrm{mol}$

Figura 2. Estructura química de las chalconas 40, 42 y 43 


\section{Estudio de toxicidad subcrónica}

Se observaron los animales individualmente al menos una vez durante los primeros 30 minutos después de la administración por vía IP, periódicamente durante las primeras 24 horas y luego diariamente durante 13 días después de la última administración (hasta el día 34), excepto cuando se requirió que sean retirados del estudio por razones de bienestar animal o se encontrasen muertos.

\section{Peso corporal y coeficiente de órganos}

Se eutanasiaron los animales de acuerdo con el protocolo de eutanasia del Comité Institucional de Ética para Uso de Animales (CIEA) de la Universidad Peruana Cayetano Heredia. Se extirparon y pesaron el hígado, el bazo, los riñones y el cerebro. Además, se incluyeron secciones del intestino. Se determinó el peso del órgano en relación con el peso corporal (coeficiente de órgano).

\section{Determinación de parámetros bioquímicos}

Antes de ser eutanasiados, se trasladó a los animales a jaulas, sin alimento por seis horas, antes de la toma de muestras de sangre por punción cardiaca. El suero se obtuvo por centrifugación a $3000 \mathrm{~g}$ durante $10 \mathrm{~min}$. Los parámetros medidos incluyeron los niveles de dos enzimas indicadoras de los efectos hepatocelulares: aspartato aminotransferasa (AST/GOT) y alanina aminotransferasa (ALT/GPT); creatinina, proteína $\mathrm{C}$ reactiva y glucosa. Se determinaron estos parámetros bioquímicos utilizando kits comerciales (Wiener lab., Argentina). Estos kits comerciales no precisan valores de referencia para animales. El análisis se realizó comparando los grupos tratados con el grupo control.

\section{Histopatología}

Se embebieron los órganos en parafina; luego, se tiñeron secciones de $4 \mu \mathrm{m}$ con hematoxilina-eosina utilizando técnicas estándares. El análisis de cada muestra por microscopía óptica se realizó en cinco campos a un aumento de 20×. El patólogo que realizó la histopatología desconocía la identidad de las muestras. Los campos se seleccionaron de forma aleatoria y continua. La evaluación de dicho material se realizó de forma cualitativa ${ }^{(4)}$, donde: ausente $=0$, leve $=+$, moderado $=++$ y severo $=+++$. Se registraron las siguientes características histopatológicas: edema, inflamación y necrosis.

\section{Análisis estadístico}

Para determinar la distribución normal de los datos se empleó el gráfico de probabilidad normal (QQ plot). Dado este cumplimiento, la comparación de los datos (peso, coeficiente de órganos y valores bioquímicos) entre los grupos de estudio se realizó con ANOVA. Para identificar el valor de significancia entre los grupos se aplicó la comparación múltiple de medias de acuerdo con el criterio de Tukey. Para las comparaciones de los hallazgos histológicos (edema, inflamación y necrosis) entre los grupos se aplicaron las pruebas de Chi cuadrado y de Fisher. Se utilizó el programa R versión 3.3.2 para Windows. Se consideró un nivel de significancia de 0,05 .

\section{Criterios éticos}

Los procedimientos fueron aprobados por el Comité Institucional de Ética para Uso de Animales (CIEA) de la Universidad Peruana Cayetano Heredia (aprobación N. ${ }^{\circ} 0350119$, mayo de 2019 y renovada en mayo de 2021, constancia N. $\left.{ }^{\circ} 0130521\right)$.

\section{RESULTADOS}

\section{$\mathrm{DL}_{50}$ según el método Dixon's Up-and-Down}

Para determinar la $\mathrm{DL}_{50}$ se empleó un animal por dosis y por chalcona. Se inició la administración oral de chalconas (40, 42 y 43) a una dosis de $175 \mathrm{mg} / \mathrm{kg}$ de peso a un animal. Durante las primeras 48 horas de evaluaciones cualitativas del comportamiento, características neurológicas y autonómicas, no se observó letalidad, convulsiones, sedación, excitación, incoordinación motora, agresividad, marcha anormal, saltos, retorcimientos abdominales, piloerección ni estereotipias (olfateo, masticación o movimientos de la cabeza). Los animales sobrevivieron luego del periodo de observación; por lo tanto, se procedió a administrar la siguiente dosis de $550 \mathrm{mg} / \mathrm{kg}$ de peso.

De igual manera que en la anterior dosis, los animales tratados (chalconas 40, 42 y 43) no mostraron evidencia de alteración en el comportamiento, características neurológicas y autonómicas durante los seis días de observación. En la necropsia se observó: chalcona 40, esplenomegalia; chalcona 42, órganos sin alteraciones; chalcona 43, alteraciones anatómicas como sufrimiento visceral y edema (en intestino e hígado), formación de fibrina en hígado, fusión de hígado con diafragma y formación de ligamentos entre las paredes externas del intestino. Estas alteraciones por efecto de la chalcona 43 se observaron en los animales que recibieron ambas dosis (175 mg/kg y $550 \mathrm{mg} / \mathrm{kg}$ de peso).

\section{Estudio de toxicidad subcrónica}

Todos los animales luego de la segunda administración presentaron un breve letargo (30 minutos) y dolor en la zona abdominal. Pasado ese periodo, los animales retomaron su actividad regular. Además, fue posible evidenciar cambios en el comportamiento de los ratones (agresividad) desde finalizada la aplicación del tratamiento (día 21) con la chal- 
cona $43(20 \mathrm{mg} / \mathrm{kg}$ y $40 \mathrm{mg} / \mathrm{kg})$ hasta la culminación del experimento (día 34). La agresividad no fue evidente en los animales tratados con las chalconas 40 y 42 a las dos dosis administradas hasta el final del experimento. En todos los grupos tratados con las chalconas no se evidenció cambio aparente en el pelo ni cambios en los ojos.

A las 24 horas de administrada la primera dosis en el grupo tratado con la chalcona 43 (40 mg/kg), los animales presentaron mucosidad en las heces. Esta manifestación continuó hasta el día 34. Por otro lado, en los grupos tratados con la chalcona 42 y con la chalcona $40(20 \mathrm{mg} / \mathrm{kg}$ y $40 \mathrm{mg} /$ $\mathrm{kg}$ ), la mucosidad se presentó entre la aplicación 4 (día 8) y la 6 (día 11). Para el término del tratamiento, los animales del grupo control presentaron el mismo signo. No se evidenciaron signos de colitis en los ratones con mucosidad en las heces. En la Figura 3 se observan los resultados del peso ganado en los animales al final del tratamiento con respecto al día 1 (inicio del tratamiento). Los grupos tratados con la chalcona 42 (40 mg/kg) o la chalcona $43(20 \mathrm{mg} / \mathrm{kg}$ y $40 \mathrm{mg} /$ $\mathrm{kg}$ ) no presentaron un aumento de peso al día 21 de experimentación en comparación con el grupo control ( $\mathrm{p}>0,05)$. Sin embargo, sí existió una diferencia estadísticamente significativa en el aumento de peso en los grupos $42(40 \mathrm{mg} / \mathrm{kg})$ $(\mathrm{p}=0,037)$ y $43(20 \mathrm{mg} / \mathrm{kg})(\mathrm{p}=0,033)$ en comparación con el grupo Sham. Dos semanas después de finalizado el trata- miento (día 34), todos los grupos tratados presentaron un modesto aumento de peso, a excepción de los grupos $40 \mathrm{y}$ 42, ambos a la dosis de $40 \mathrm{mg} / \mathrm{kg}$.

Los resultados del coeficiente de órganos (Figura 4) mostraron que las chalconas afectaron los órganos internos evaluados. La chalcona 42 a la dosis de $20 \mathrm{mg} / \mathrm{kg}$ (42A) redujo el coeficiente del bazo $(\mathrm{p}=0,034)$, mientras que la chalcona 43 , a la dosis de $40 \mathrm{mg} / \mathrm{kg}$ (43B), incrementó el coeficiente del riñón $(p=0,037)$ y el coeficiente del cerebro $(p=0,008)$. No se observaron diferencias significativas en el hígado.

Se evidenció una disminución de creatinina por efecto del tratamiento con las chalconas 42 y 43 , ambas administradas a la dosis de $20 \mathrm{mg} / \mathrm{kg}$, en comparación con el grupo control (promedio \pm desviación estándar: $2,20 \pm 1,31$; $\mathrm{p}=0,026$ y $2,50 \pm 0,74 ; \mathrm{p}=0,033$ contra $16,30 \pm 2,09 \mathrm{mg} / \mathrm{L}$, respectivamente). No se encontraron variaciones significativas en los niveles de transaminasas GOT y GPT influenciadas por el tratamiento (Figura 5). No se detectó proteína $\mathrm{C}$ reactiva en ninguna muestra.

Todos los grupos presentaron un órgano de, al menos, un ratón con una alteración histológica evaluada (nivel leve/ moderado en los grupos control y Sham; moderado/severo en los grupos tratados). No se evidenció en todos los grupos (incluido el control) necrosis en hígado, bazo, riñón y cerebro; tampoco inflamación en hígado ni bazo (Figura 6). Cabe

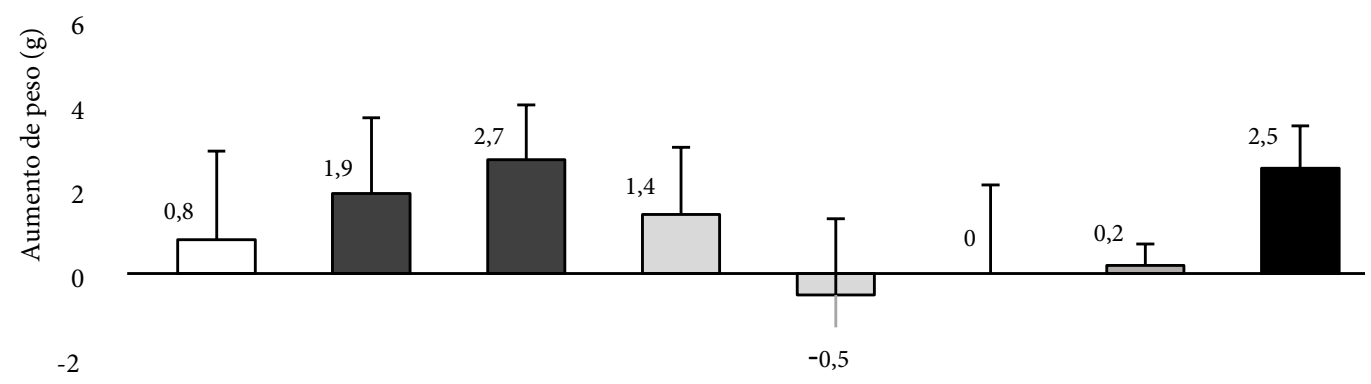

B Día 34
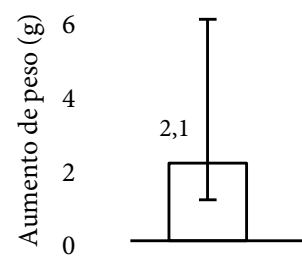

Control

$40 \mathrm{~A}$
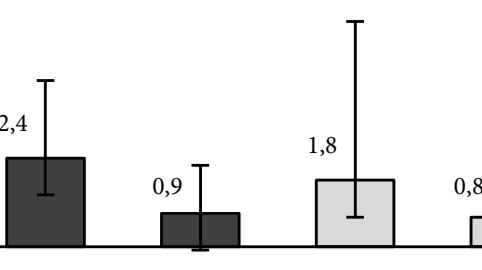

$-2$

40B

$42 \mathrm{~A}$

$42 \mathrm{~B}$

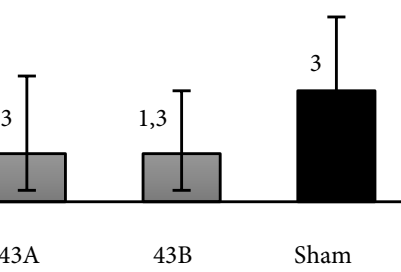

Figura 3. Aumento de peso de animales controles versus tratados con chalconas sintéticas 40, 42 y 43 (donde A: $20 \mathrm{mg} / \mathrm{kg}$ y B: $40 \mathrm{mg} / \mathrm{kg}$ ) al término del tratamiento con respecto al día 1 (inicio del tratamiento). A: día 21. B: día 34. ( $\mathrm{n}=6$ por grupo). Los datos se expresan como promedio y desviación estandar. 

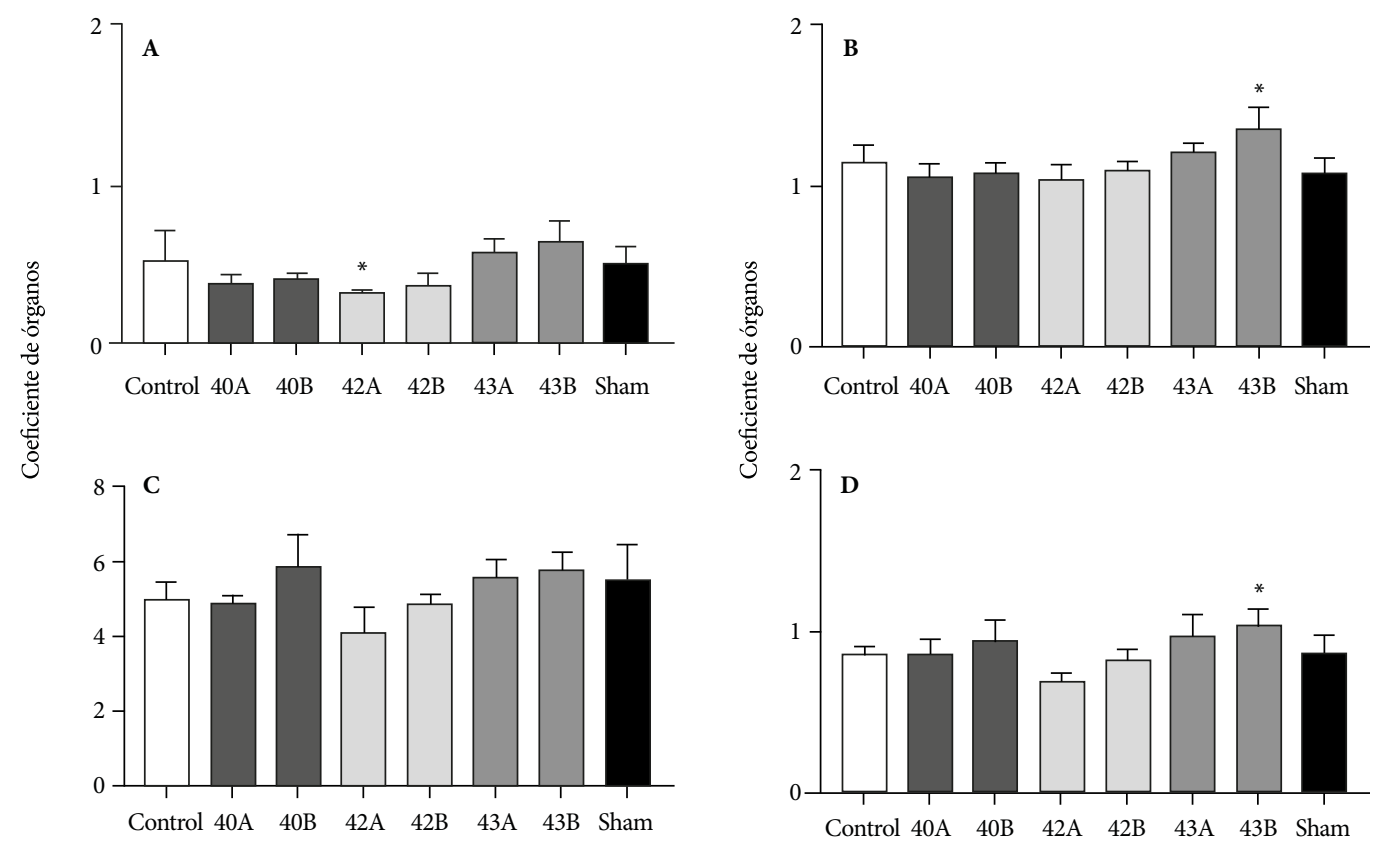

Figura 4. Coeficiente de órganos procedentes de ratones BALB/c tratados con chalconas sintéticas 40, 42 y 43 (donde A: $20 \mathrm{mg} / \mathrm{kg}$ y B: $40 \mathrm{mg} / \mathrm{kg}$ ). A: bazo, B: cerebro, C: hígado, D: riñón. Control: diluyente de chalconas. Sham: animales sin tratamiento. Los datos se expresan como promedio y desviación estandar (6 por grupo). ${ }^{*} \mathrm{p}<0,05$ comparado con el grupo control, utilizando ANOVA.

resaltar que en los intestinos de los ratones tratados (principalmente con la chalcona 43), las alteraciones histológicas fueron severas. A pesar de las alteraciones histológicas, no hubo diferencia estadísticamente significativa $(\mathrm{p}>0,05)$ entre los grupos tratados con las chalconas en comparación con el control y el grupo Sham.
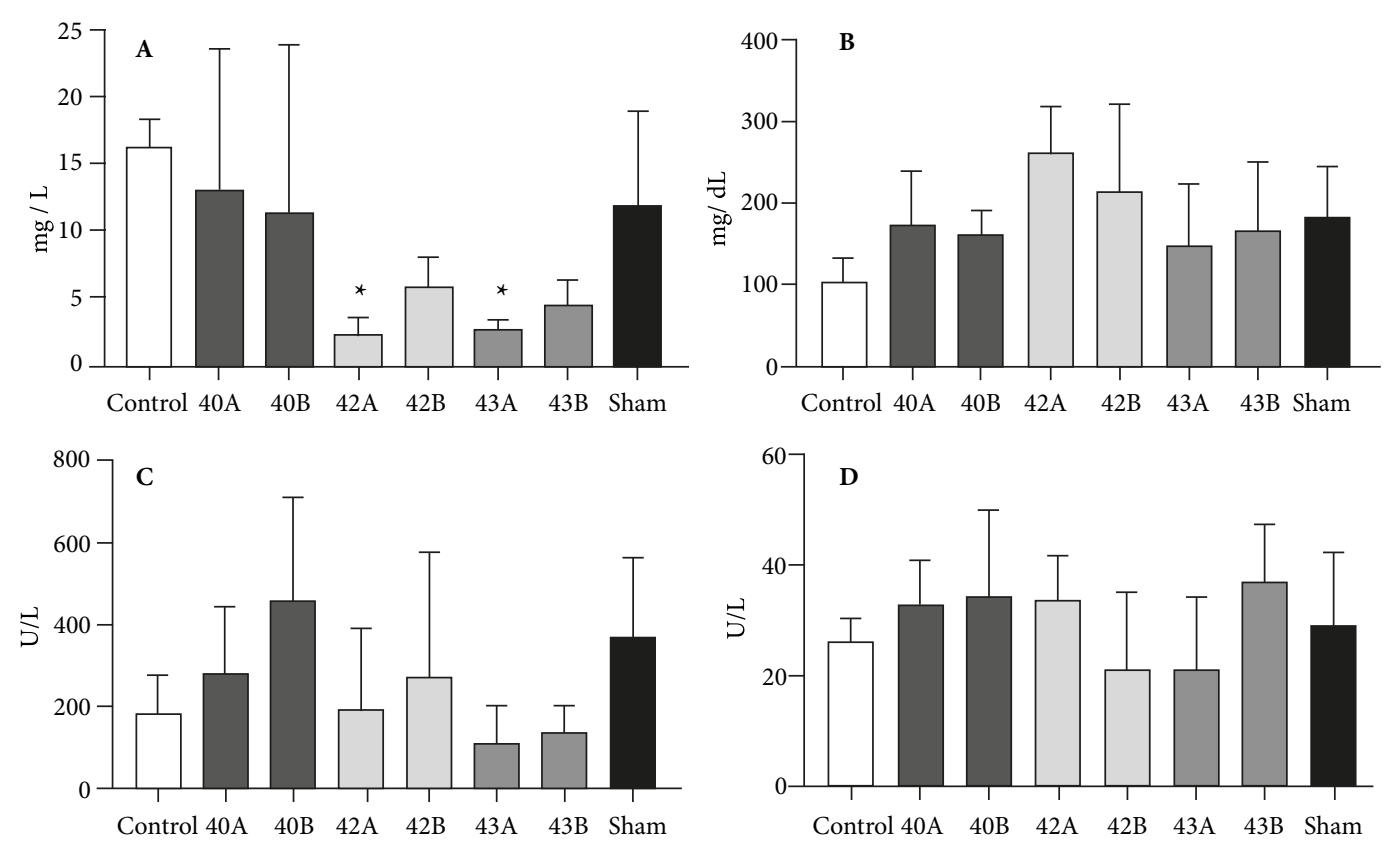

Figura 5. Análisis de parámetros bioquímicos en suero de ratones BALB/c machos tratados con chalconas sintéticas 40,42 y 43 (donde A: $20 \mathrm{mg} / \mathrm{kg}$ y B: $40 \mathrm{mg} / \mathrm{kg}$ ). Control: diluyente de chalconas. Sham: animales sin tratamiento. A: creatinina, B: glucosa, C: GOT, D: GPT. Los datos se expresan como promedio y desviación estandar (6 por grupo). ${ }^{\star} \mathrm{p}<0,05$ comparado con el grupo control, utilizando ANOVA. 
Bazo

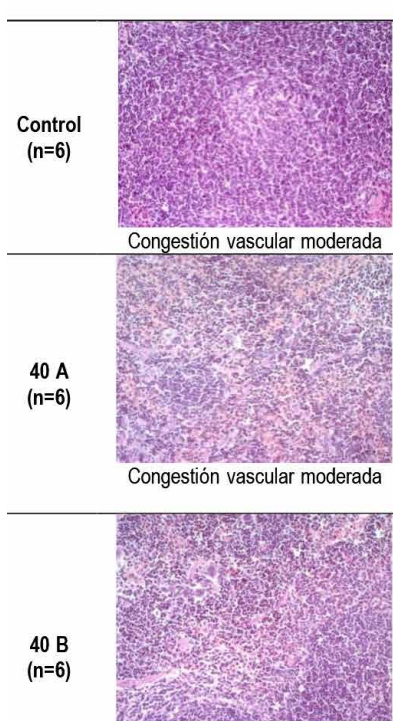

Sin edema
Riñón
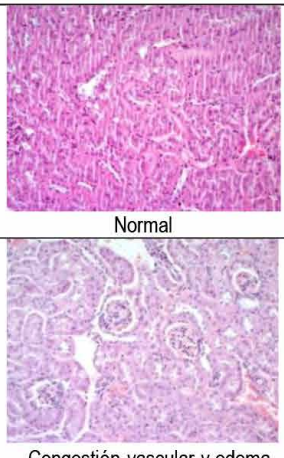

Congestión vascular y edema

leve

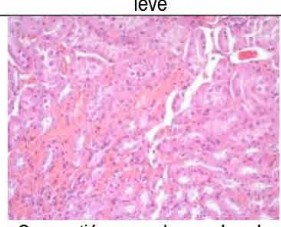

Congestión vascular moderada
Hígado

Cerebro

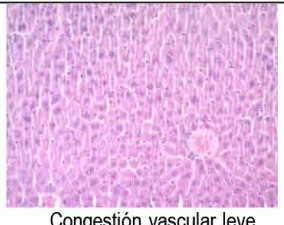

Congestión vascular leve

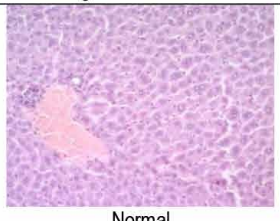

Normal

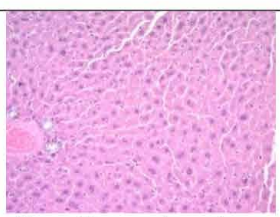

Congestión vascular y edema

leve

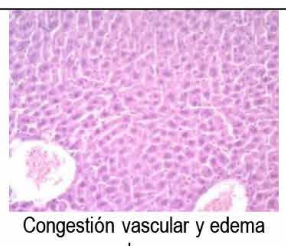

leve

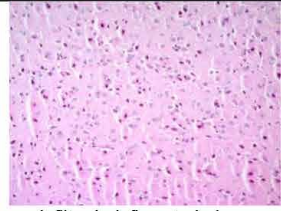

Infiltrado inflamatorio leve

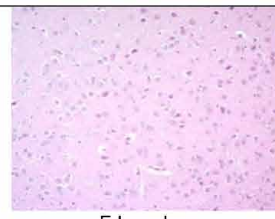

Edema leve

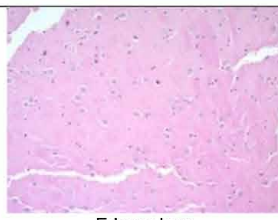

Edema leve

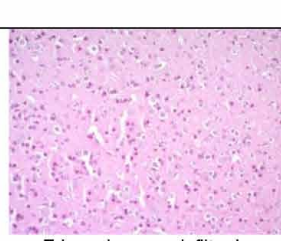

Edema leve con infiltrado inflamatorio

Intestino

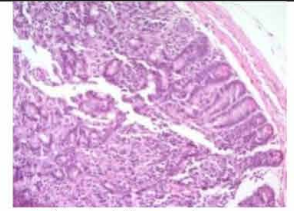

Infiltrado inflamatorio severo

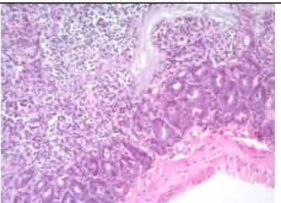

Infiltrado inflamatorio severo con destrucción de criptas; necrosis

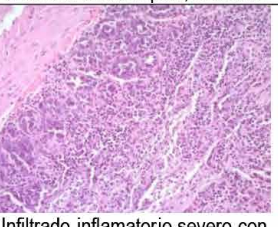

Infiltrado inflamatorio severo con destrucción de arquitectura normal con necrosis

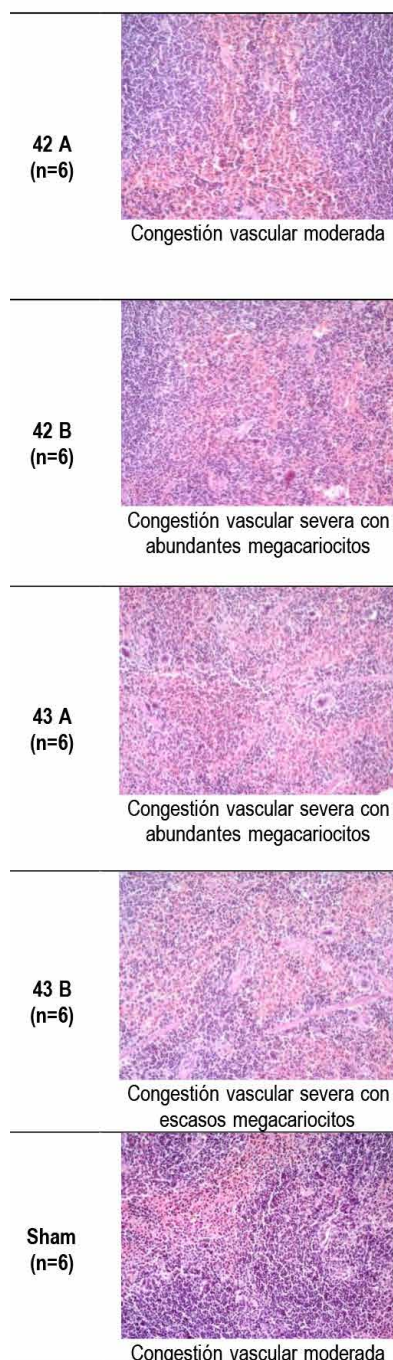

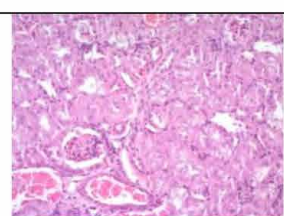

leve

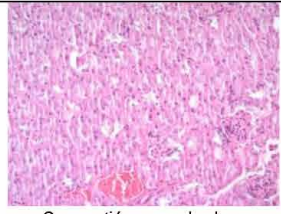

Congestión vascular leve

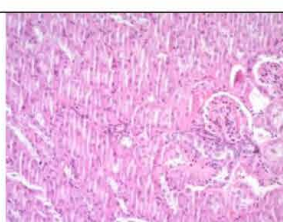

Edema leve

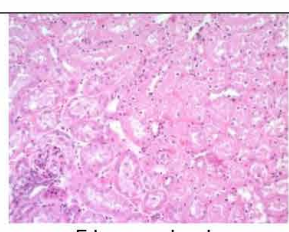

Edema moderado

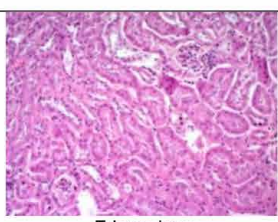

Edema leve
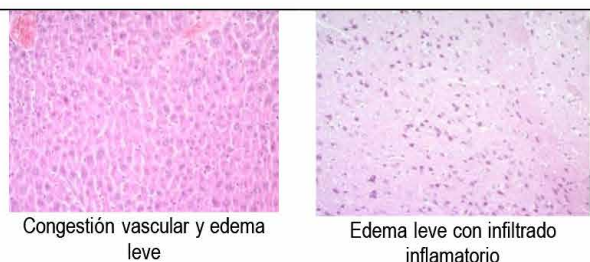

inflamatorio

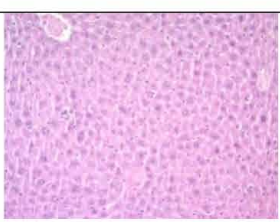

Congestión vascular y edema leve
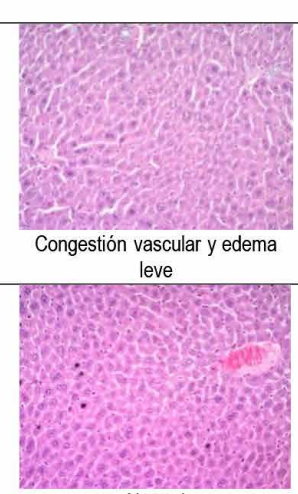

Normal

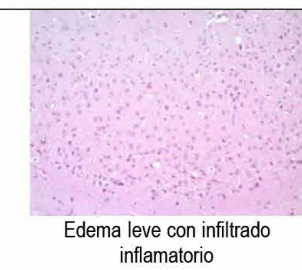

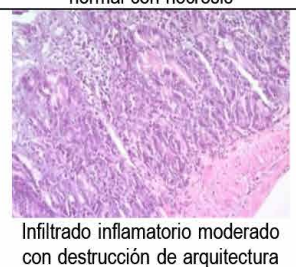

con destrucción de arquitectura normal con necrosis

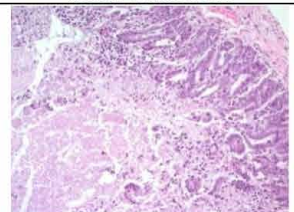

Infiltrado inflamatorio moderado con destrucción de arquitectura normal con necrosis

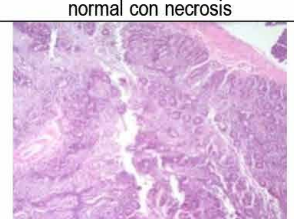

Infiltrado inflamatorio moderado con destrucción de arquitectura normal

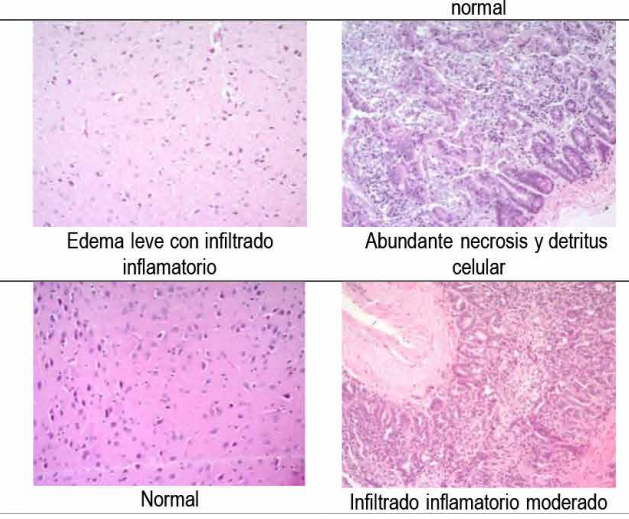

Figura 6. Imágenes representativas de la evaluación histopatológica de órganos vitales de ratón en el estudio de toxicidad subcrónica de las chalconas sintéticas. Secciones representativas de bazo, riñón, hígado, cerebro e intestino teñidas con hematoxilina-eosina, tomadas de los ratones sin tratamiento (Sham), control (solventes), administrados con $20 \mathrm{mg} / \mathrm{kg}$ de chalconas sintéticas (40A, 42A y 43A) o $40 \mathrm{mg} / \mathrm{kg}$ de chalconas sintéticas (40B, 42B y 43B). Aumento de 200×. 


\section{DISCUSIÓN}

En el presente estudio se evaluó la toxicidad in vivo subcrónica por vía IP de las chalconas sintéticas 40,42 y 43 siguiendo el esquema de tratamiento referencial para las leishmaniosis; así como la $\mathrm{DL}_{50}$, por vía oral. Las tres chalconas, a la dosis de $40 \mathrm{mg} / \mathrm{kg}$, no alteraron los parámetros bioquímicos en suero. De otro lado, algunas de las características anátomo-histopatológicas de los órganos principales, coeficientes de órganos y signos del comportamiento en los animales resultaron alterados, principalmente en aquellos tratados con la chalcona 43 .

Pocos estudios han reportado el análisis de la toxicidad in vivo de chalconas y su proceso ADME (absorción, distribución, metabolismo y excreción) en el organismo, a pesar de su estructura química sencilla y promisorios efectos a nivel de la actividad biológica. Estos estudios han evaluado principalmente la actividad biológica en diferentes modelos patológicos ${ }^{(2-4,6,9,14,15)}$. Sobre la base de los resultados del presente estudio, se sugiere abordar los procesos ADME principalmente con las chalconas 40 y 42 en futuros estudios.

Según el modelo in vivo de leishmaniosis, la vía de administración, el esquema del tratamiento y el efecto biológico pueden diferir. De Mello et al. determinaron la no toxicidad de dos chalconas sintéticas (por vía intralesional y por vía IP) evaluadas hasta una dosis de $10 \mathrm{mg} / \mathrm{kg}$ de peso corporal administradas tres veces por semana durante cuatro semanas y la actividad antiparasitaria de las mismas contra Leishmania braziliensis en hámsteres dorados ${ }^{(16)}$. Aponte et al. observaron una alta actividad in vivo de las chalconas $42 \mathrm{y}$ 43 (administradas por vía intralesional) en el modelo murino infectado por Leishmania amazonensis en la almohadilla plantar ${ }^{(9)}$. Tomando en consideración lo anterior, se valida la importancia de la selección de la mejor vía de administración cuando los compuestos están concebidos para ser aplicados como tratamiento, de tal forma que se favorezca la biodisponibilidad y se maximice su actividad biológica.

La administración por vía IP de fármacos se emplea ampliamente en roedores porque facilita la velocidad de absorción en comparación con las vías intramuscular y subcutánea ${ }^{(17,18)}$. La principal limitación de la vía IP es el efecto del metabolismo inicial; sin embargo, se considera que los fármacos de bajo peso molecular (como las chalconas) administrados por vía IP conducen a una menor exposición sistémica ${ }^{(17)}$. Otras limitaciones son la esterilidad y la irritabilidad que podrían generar ${ }^{(17)}$. Los compuestos irritantes pueden causar íleo e inflamación peritoneal, lo que podría favorecer el desarrollo de adherencias, características que fueron evidentes en la necropsia de los animales tratados con la chalcona 43. A pesar de que la vía de administración difiere (vía oral, $1000 \mathrm{mg} / \mathrm{kg} / \mathrm{día}$ ) de la empleada en este estudio, se ha informado que otras chalconas pueden afectar algunos parámetros fisiológicos, como el peso de los riñones, alterando el coeficiente del órgano, y generar cambios macroscópicos mínimos en el hígado ${ }^{(19)}$, tal como se ha registrado en los animales del grupo chalcona 43B $(40 \mathrm{mg} / \mathrm{kg}$ ) en este estudio. En contraste, otros autores no han reportado cambios histológicos en riñón o en hígado, ni la alteración de parámetros bioquímicos generados por chalconas administradas por vía intralesional ${ }^{(15)}$.

La absorción de los fármacos que se aplican en la cavidad peritoneal se debe a la difusión a los tejidos circundantes (entre ellos el intestino) ${ }^{(17,18)}$. En el presente estudio, proponemos que la administración IP y la difusión intestinal de las chalconas pueden generar el aumento de moco en las heces, como barrera biológica, principalmente en los animales tratados con la chalcona 43 , siendo esto confirmado a nivel histopatológico. En cuanto a la relevancia toxicológica de la interacción entre el moco en las heces y los compuestos xenobióticos para el huésped, la información es limitada. Los estudios señalan que la cantidad de moco intestinal puede aumentar o disminuir según el compuesto químico administrado o por la dieta ${ }^{(20,21)}$. Aún es poco conocido cómo los químicos ambientales (plaguicidas, entre otros) pueden interactuar con el moco intestinal y las bacterias «mucofílicas» de la microbiota ${ }^{(20)}$.

Es de esperar que, a pesar de los factores ambientales, los coeficientes de órganos para una misma cepa de ratón sean semejantes. Los coeficientes de órganos del grupo control determinados en el presente estudio para la cepa $\mathrm{BALB} / \mathrm{c}$ son semejantes a los previamente reportados por Shi et al. para su grupo control ${ }^{(22)}$. Comúnmente, las alteraciones anormales del peso corporal en estudios de toxicidad se consideran indicadores críticos de los efectos adversos. Los compuestos administrados y el dolor abdominal pueden influir en el apetito de los animales y por consecuencia en el peso. Sin embargo, en el presente estudio no existió una diferencia significativa en el peso ganado entre el grupo control y los grupos tratados al finalizar el tratamiento (día 21). El aumento de peso registrado en nuestro estudio es semejante a lo reportado en ratones por Zhao et al. ${ }^{(23)} \mathrm{y}$ Shi et al. ${ }^{(22)}$.

La determinación de la toxicidad subcrónica en el presente estudio evidenció cambios en el comportamiento de los animales tratados con la chalcona $43(40 \mathrm{mg} / \mathrm{kg})$. A pesar de que modulaciones en el comportamiento (sistema nervioso) debidas a derivados de chalconas se reportaron previamente ${ }^{(24)}$; en nuestro estudio, la agresividad de los animales no se validó con los análisis histopatológicos efectuados.

Teniendo en cuenta que las condiciones de laboratorio, tales como alimentación, horarios de exposición a la luz, disposición de alimento y agua, varían entre las instituciones, así como los métodos que se emplean en la cuantificación de los parámetros bioquímicos, se hace compleja la generalización de los valores de referencia. Mazzaccara et al. sugieren que se empleen parámetros hematológicos dependientes del sexo y la edad para 
cada cepa de ratón, ya que estos varían de una cepa a otra ${ }^{(25)}$. Aunque hay artículos en la literatura que discuten los parámetros hematológicos y bioquímicos para ratones BALB/c sanos, pocos abordan la evaluación de estos parámetros en situaciones patológicas (26); además, estos han sido obtenidos empleando diferentes metodologías. Según la literatura, se sugiere comparar los parámetros bioquímicos entre los grupos tratados y los grupos sin tratamiento. Considerando los acercamientos por obtener valores referenciales de parámetros hematológicos en ratones machos $\mathrm{BALB} / \mathrm{c}$, los valores de GPT y GOT hallados en el presente estudio son comparables a los establecidos por Lima et al. ${ }^{(27)}$ y Fernandes et al. ${ }^{(28)}$. No obstante, los valores reportados para creatinina en ambos estudios no son comparables. Los resultados de creatinina en el presente estudio se encuentran por debajo de los reportados por Lima et al. $(0,58 \pm 0,24 \mathrm{mg} / \mathrm{dL})^{(27)}$. Por otro lado, SilvaSantana et al. reportan valores referenciales no detectables para creatinina $(<0,2 \mathrm{mg} / \mathrm{dL})$, determinados con el espectrofotómetro automático VITROS ${ }^{\circledR} 35$ (Ortho Clinical Diagnostics) ${ }^{(29)}$. Para un tamizaje de mayor sensibilidad se sugiere incluir otros parámetros (fosfatasa alcalina, triglicéridos, nitrógeno ureico en sangre, entre otros), que puedan complementar la detección de alteraciones en el metabolismo de los animales.

Según el método de Dixon, se recomienda continuar el ensayo con dosis superiores a $550 \mathrm{mg} / \mathrm{kg}$ de peso. Dada la disponibilidad limitada de los compuestos sintéticos en este estudio, esto no fue posible. Cabe señalar que la dosis máxima administrada de las chalconas $(550 \mathrm{mg} / \mathrm{kg}$ de peso, por vía oral) fue 100 veces más alta que la dosis que probó ser efectiva (5 mg/kg/día, 8 inoculaciones por vía intralesional) en la reducción significativa de la carga parasitaria en el modelo de ratón infectado por L. amazonensis en la almohadilla plantar ${ }^{(9)}$.

\section{REFERENCIAS BIBLIOGRÁFICAS}

1. Nowakowska Z. A review of anti-infective and anti-inflammatory chalcones. Eur J Med Chem. 2007;42(2):125-37. doi: 10.1016/j.ejmech.2006.09.019.

2. Li W, Xu F, Shuai W, Sun H, Yao H, Ma C, et al. Discovery of Novel Quinoline-Chalcone Derivatives as Potent Antitumor Agents with Microtubule Polymerization Inhibitory Activity. J Med Chem. 2019;62(2):993-1013. doi: 10.1021/acs.jmedchem.8b01755.

3. Aponte JC, Verástegui M, Málaga E, Zimic M, Quiliano M, Vaisberg AJ, et al. Synthesis, cytotoxicity, and anti-Trypanosoma cruzi activity of new chalcones. J Med Chem. 2008;51(19):6230-4. doi: 10.1021/jm800812k.

4. de Mello TF, Bitencourt HR, Pedroso RB, Aristides SM, Lonardoni MV, Silveira TG. Leishmanicidal activity of synthetic chalcones in Leishmania (Viannia) braziliensis. Exp Parasitol. 2014;136:27-34. doi: 10.1016/j. exppara.2013.11.003.

5. Boeck P, Bandeira Falcão CA, Leal PC, Yunes RA, Filho VC, Torres-Santos $\mathrm{EC}$, et al. Synthesis of chalcone analogues with increased antileishmanial activity. Bioorg Med Chem. 2006;14(5):1538-45. doi: 10.1016/j. bmc.2005.10.005

6. García AR, Oliveira DMP, Jesus JB, Souza AMT, Sodero ACR, Vermelho $\mathrm{AB}$, et al. Identification of Chalcone Derivatives as Inhibitors of Leishmania infantum Arginase and Promising Antileishmanial Agents. Front Chem. 2021;8:624678. doi: 10.3389/fchem.2020.624678.
Empleando el software predictor de propiedades químicas admetSAR ${ }^{\mathrm{TM}}$ versión $2.0^{(30)}$, se pudo determinar in silico la $\mathrm{DL}_{50}$ por vía oral en ratas: chalcona $40(5998,06 \mathrm{~g} / \mathrm{kg})$, chalcona 42 (10 986,05 g/kg) y chalcona $43(9445,40 \mathrm{~g} / \mathrm{kg})$.

En conclusión, los hallazgos generales indican que la $\mathrm{DL}_{50}$ para las tres chalconas es superior a $550 \mathrm{mg} / \mathrm{kg}$ de peso. Las chalconas 40 y 42 son relativamente no tóxicas, por lo que pueden considerarse para una aplicación segura en ratones $\mathrm{BALB} / \mathrm{c}$. Consideramos de relevancia extender la exploración de la toxicidad a análogos de chalconas que muestren actividad antileishmanial y sean lead compounds candidatos para encaminar el desarrollo de nuevos fármacos para leishmaniosis. Tales estudios deberán tomar en cuenta la evaluación de la toxicidad, la vía de administración y el proceso ADME en modelos in vivo. De esta manera se contribuirá con los esfuerzos de la comunidad científica ${ }^{(4,6,9,16)}$ en la validación de la actividad biológica de las chalconas en modelos murinos de leishmaniosis cutánea y visceral.

Agradecimientos: Los autores agradecen a Luis Cano Ayestas, M. D., $\mathrm{Ph}$. D., especialista en anatomía patológica, por el análisis histopatológico.

Contribuciones de autoría: KC, JA, MS, VA y DC concibieron y diseñaron el estudio; KC y CY recopilaron los datos; IC y VJ contribuyeron a la recopilación de datos y tuvieron a cargo la síntesis de las chalconas; KC, MS, VA y DC participaron en el procesamiento, análisis e interpretación de los datos; $\mathrm{KC}$ fue la principal responsable de la redacción del manuscrito; MS, VA y DC revisaron críticamente el manuscrito. Todos los autores aprobaron la versión final y son responsables del contenido del manuscrito.

Financiamiento: Fondo Nacional de Desarrollo Científico, Tecnológico y de Innovación Tecnológica del Consejo Nacional de Ciencia Tecnología e Innovación Tecnológica (CONCYTEC) (Convenio C-1122015 FONDECYT).

Conflictos de interés: Los autores declaran no tener conflictos de interés.
7. Van Voorhis WC, Adams JH, Adelfio R, Ahyong V, Akabas MH, Alano $\mathrm{P}$, et al. Open Source Drug Discovery with the Malaria Box Compound Collection for Neglected Diseases and Beyond. PLoS Pathog. 2016;12(7):e1005763. doi: 10.1371/journal.ppat.1005763.

8. Ghorbani M, Farhoudi R. Leishmaniasis in humans: drug or vaccine therapy?. Drug Des Devel Ther. 2017;12:25-40. doi: 10.2147/DDDT.S146521.

9. Aponte JC, Castillo D, Estevez Y, Gonzalez G, Arevalo J, Hammond GB, et al. In vitro and in vivo anti-Leishmania activity of polysubstituted synthetic chalcones. Bioorg Med Chem Lett. 2010;20(1):100-3. doi: 10.1016/j. bmcl.2009.11.033.

10. Organisation for Economic Cooperation and Development. Test No. 425: Acute Oral Toxicity: Up-and-Down Procedure, OECD Guidelines for the Testing of Chemicals, Section 4. París: OECD Publishing; 2008. doi: 10.1787/9789264071049-en.

11. Dixon WJ. The Up-and-Down method for small samples. J Am Stat Assoc. 1965;60(312):967-78. Disponible en: https://doi.org/10.2307/2283398.

12. Du Z, She X, Ma J, Wang Z, Wu H, Li Y, et al. A facile synthetic route to two chalcones. Journal of Chemical Research. 2004;2004(1):45-6. doi: 10.3184/030823404323000756.

13. Roux S, Sablé E, Porsolt RD. Primary observation (Irwin) test in rodents for assessing acute toxicity of a test agent and its effects on behavior and physiological function. Curr Protoc Pharmacol. 2005;Chapter 10:Unit 10.10. doi: 10.1002/0471141755.ph1010s27. 
14. Staurengo-Ferrari L, Ruiz-Miyazawa KW, Pinho-Ribeiro FA, Fattori V, Zaninelli TH, Badaro-Garcia S, et al. Trans-Chalcone Attenuates Pain and Inflammation in Experimental Acute Gout Arthritis in Mice. Front Pharmacol. 2018;9:1123. doi: 10.3389/fphar.2018.01123.

15. Dhiyaaldeen SM, Amin ZA, Darvish PH, Mustafa IF, Jamil MM, Rouhollahi E, et al. Protective effects of (1-(4-hydroxy-phenyl)-3-m-tolyl-propenone chalcone in indomethacin-induced gastric erosive damage in rats. BMC Vet Res. 2014;10:961. doi: 10.1186/ s12917-014-0303-7.

16. de Mello TF, Cardoso BM, Lopes SN, Bitencourt HR, Voltarelli EM, Hernandes L, et al. Activity of synthetic chalcones in hamsters experimentally infected with Leishmania (Viannia) braziliensis. Parasitol Res. 2015;114(10):3587-600. doi: 10.1007/s00436-015-4581-1.

17. Al Shoyaib A, Archie SR, Karamyan VT. Intraperitoneal Route of Drug Administration: Should it Be Used in Experimental Animal Studies? Pharm Res. 2019;37(1):12. doi: 10.1007/s11095-019-2745-x.

18. Intraperitoneal Drug Administration. En: Techniques in the Behavioral and Neural Sciences [Internet]. Elsevier; 1994 [citado el 23 de junio de 2021]; 12:46-58. Disponible en: https://linkinghub.elsevier.com/ retrieve/pii/B9780444818713500102

19. Maronpot RR. Toxicological assessment of Ashitaba Chalcone. Food Chem Toxicol. 2015;77:111-9. doi: 10.1016/j.fct.2014.12.021.

20. Gillois K, Lévêque $M$, Théodorou V, Robert H, Mercier-Bonin M. Mucus: An Underestimated Gut Target for Environmental Pollutants and Food Additives. Microorganisms. 2018;6(2):53. doi: 10.3390/ microorganisms6020053.

21 Chassaing B, Koren O, Goodrich JK, Poole AC, Srinivasan S, Ley RE, et al. Dietary emulsifiers impact the mouse gut microbiota promoting colitis and metabolic syndrome. Nature. 2015;519(7541):92-6. doi: 10.1038 /nature14232

22. Shi JP, Li SX, Ma ZL, Gao AL, Song YJ, Zhang H. Acute and sub-chronic toxicity of tetrandrine in intravenously exposed female BALB/c mice. Chin J Integr Med. 2016;22(12):925-931. doi: 10.1007/s11655-015-2303-2.
23. Zhao Q, Yang M, Deng Y, Yu H, Wang L, Teng F, et al. The Safety Evaluation of Salvianolic Acid B and Ginsenoside Rg1 Combination on Mice. Int J Mol Sci. 2015;16(12):29345-56. doi: 10.3390/ijms161226176.

24. Kwak J, Kim MJ, Choi KC, Choi HK, Jun W, Park HJ, et al. The chalcone derivative Chana 1 protects against amyloid $\beta$ peptide-induced oxidative stress and cognitive impairment. Int J Mol Med. 2012;30(1):193-8. doi: 10.3892/ijmm.2012.984.

25. Mazzaccara C, Labruna G, Cito G, Scarfò M, De Felice M, Pastore L, et al. Age-Related Reference Intervals of the Main Biochemical and Hematological Parameters in C57BL/6J, 129SV/EV and C3H/HeJ Mouse Strains. PLoS One. 2008;3(11):e3772. doi: 10.1371/journal.pone.0003772.

26. Barbosa BDS, Praxedes ÉA, Lima MA, Pimentel MML, Santos FA, Brito PD, et al. Haematological and Biochemical Profile of Balb-c Mice. Acta Sci Vet. 2017;45(1):5. doi: 10.22456/1679-9216.80473.

27. Lima CM, Lima AK, Melo MGD, Dória GAA, Leite BLS, Serafini $\mathrm{MR}$, et al. Valores de referência hematológicos e bioquímicos de ratos (Rattus novergicus linhagem Wistar) provenientes do biotério da Universidade Tiradentes. Sci Plena. 2014;10(034601):1-9. Disponible en: https://www.scientiaplena.org.br/sp/article/view/1784/948

28. Fernandes DP, Pimentel MML, Santos FAD, Praxedes ÉA, Brito PD, Lima MA, et al. Hematological and biochemical profile of BALB/C nude and C57BL/6 SCID female mice after ovarian xenograft. An Acad Bras Cienc. 2018;90(4):3941-3948. doi: 10.1590/00013765201820180586.

29. Silva-Santana G, Bax JC, Fernandes DCS, Bacellar DTL, Hooper C, Dias AASO, et al. Clinical hematological and biochemical parameters in Swiss, BALB/c, C57BL/6 and B6D2F1 Mus musculus. Animal Model Exp Med. 2020;3(4):304-315. doi: 10.1002/ame2.12139.

30. Yang H, Lou C, Sun L, Li J, Cai Y, Wang Z, et al. admetSAR 2.0: web-service for prediction and optimization of chemical ADMET properties. Bioinformatics. 2019;35(6):1067-1069. doi: 10.1093/bioinformatics/ bty707. 